\title{
A Clinical Study of Epidemiology of Anterior Uveitis in a Tertiary Care Teaching Hospital
}

\section{Dawson Vinay Kumar ${ }^{1}$, Dawson Elizabeth Manasvi²}

Section: Healthcare

Sci. Journal Impact

Factor: 6.1 (2018)

ICV: 90.90 (2018)

cc) (4) (8)

Copyright@IJCRR

'Associate Professor, Department of Ophthalmology, Rajiv Gandhi Institute of Medical Sciences [RIMS], Adilabad-504001, Telangana State, India; ${ }^{2} \mathrm{PG}$, Department of Biochemistry, Nizams Institute of Medical Sciences, Hyderabad-500082, Telangana State, India.

\section{ABSTRACT}

Introduction: Uveitis, is a complex intraocular inflammatory disease with multifactorial etiology. The cause of inflammation might be an infectious agent or trauma, but in some cases, the underlying mechanism appears to be autoimmune.

Aim: The present study aimed to evaluate the etiological pattern, treatment, and outcome and complications of anterior uveitis.

Methods: A prospective clinical study was done in the Department of Ophthalmology. All patients aged between 21-70 yrs with clinical signs and symptoms of anterior uveitis were included. All the patients underwent complete clinical evaluation and investigation were performed to determine etiology. Patients were put on specific and non-specific treatment and were followed up for 6 months. Complications were noted.

Results: Patients $n=44(88 \%)$ of the total $n=50(100 \%)$ patients were having acute anterior uveitis and $n=6(12 \%)$ were having a chronic type of anterior uveitis shown in table 3 . In the present study, most of the cases $n=45(90 \%)$ were suffering from nongranulomatous infection and $n=5(10 \%)$ were having granulomatous inflammation. Cataract was the common compilation of anterior uveitis out of the $n=7$ cases in this study $n=6$ cases reached the maximum post-operative vision of $6 / 12$ and one was able to reach $n=6 / 9$ with refraction and they were prescribed glasses.

Conclusion: The primary etiology of anterior uveitis remained obscure in several cases. Every case should be subjected to complete clinical examination and investigations to determine the final diagnosis. Early diagnosis and treatment will ensure a good clinical outcome. However, ocular morbidity remains a problem in chronic and recurrent cases.

Key Words: Clinical Study, Etiological pattern, Anterior Uveitis, Visual impairment, Ocular inflammation

\section{INTRODUCTION}

Uveitis is a sight-threatening disease with intraocular inflammation due to varied etiology. It may lead to irreversible visual loss if not detected and treated early. Approximately $35 \%$ of patients with uveitis exhibit blindness or visual impairment in one of the eyes. ${ }^{1}$ It is the fifth most common cause of visual loss in developed countries and up to $20 \%$ of legal blindness occurs due to complications of uveitis. ${ }^{2,3}$ One of the crucial factors in the management of uveitis is the identification of correct etiological factors involved which can be infectious, autoimmune, and traumatic. Etiologies have been found to vary based on environmental factors, racial and genetic factors. The anterior uveitis is the most common type of uveitis. Clinically acute unilateral, non-infectious, and non-granulomatous forms are more frequently encountered. Idiopathic anterior uveitis is commonly found in all age groups. Traumatic anterior uveitis is commonly found in children. ${ }^{4}$ The mean age is $4^{\text {th }}$ decade and more commonly affects males as compared to females. ${ }^{5}$ The precise diagnosis of anterior uveitis is still a challenge, but the autoimmune factor seen to be involved in a large number of cases of anterior uveitis. ${ }^{6}$ The anterior uveitis is categorized as iritis, anterior cyclitis, and iridocyclitis. It is clinically presented as a red painful eye. The other complaints of patients with anterior uveitis include photophobia, tearing, and blurred vision. It has been found that in about $30 \%$ of cases of acute anterior uveitis is associated with HLA-B27 [Human leukocyte antigen B27] haplotype and the uveitis presented here is nongranulomatous. ${ }^{7}$ It is found that these patients also present with reactive arthritis, ankylosing spondylitis, inflammatory bowel disease, and psoriatic arthritis. The exact pathophysiology of HLA-B27 positive anterior uveitis is unknown it is

\section{Corresponding Author:}

Dr. Dawson Vinay Kumar, Manasvi Eye Clinic, Near Andhra Bank, Maheshwari Talkies Road, Adilabad-504001. Mobile: +918106095906; Email: drdawsoneye@yahoo.co.in

ISSN: 2231-2196 (Print)

Received: 16.07 .2020
ISSN: 0975-5241 (Online)

Revised: 09.09.2020
Accepted: 10.10 .2020

Published: 14.12 .2020 
thought that Gram-negative bacilli in the gut or chlamydia infection might play a role. Rosenbaum et al. ${ }^{8}$ in animal models found that oral antibiotics modulate the severity of experimental anterior uveitis by increasing lymphocytes in the gut and extra-intestinal tissues and by decreasing effectors T-cells and cytokines. The granulomatous uveitis occurs due to tuberculosis, sarcoidosis, and leprosy. Tuberculous anterior uveitis is presented with mutton-fat ketotic precipitates, broad posterior synechiae, and iris nodules. ${ }^{9}$ In most cases, the aqueous aspirates of acid-fast bacilli and culture remain negative. Polymerase chain reaction (PCR) for mycobacterial DNA is positive. ${ }^{10}$ Anterior uveitis due to sarcoidosis often has a similar presentation as tubercular uveitis. Elevated serum angiotensin-converting enzyme, negative tuberculin test and hilar lymphadenopathy can distinguish sarcoidosis from tuberculosis. The treatment of infectious uveitis is aimed at the causative pathogens. In non-infectious uveitis use of local steroids and immunosuppressants has gained popularity. We in the present study tried to evaluate the frequency of anterior uveitis in our cases, clinical presentations, diagnosis, treatment, and prognosis.

\section{MATERIALS AND METHODS}

\section{Study Design: Prospective study}

Department: Department of Ophthalmology

Institute: Rajiv Gandhi Institute of Medical Sciences [RIMS] Adilabad.

Ethical Permission: Institutional Ethical permission was obtained for this study wide the reference No: 99/06/RIMS/ EC/2018. Dated 31/03/2018.

Consent for participation: Written consent from all the patients for voluntary participation in the study.

\section{Sample size}

It was calculated with the following data: margin of error $\alpha=$ Alpha Error $(5 \%), 1-\beta=$ Power $(95 \%)$, the approximate population of this area is 250000 if $3 \%$ of the population is affected the required sample size is 45 we have taken $n=50$ patients in this study.

Patients aged 21-70 years with signs and symptoms of anterior uveitis were included. Excluded were patients with anterior uveitis following penetrating ocular injuries, corneal ulcers, intraocular surgeries, and those associated with posterior or pan-uveitis. A detailed history was obtained from the patients. Clinical examination included a test for visual acuity, patients were examined under a slit lamp. Details of disease severity, laterality, chronicity, and ocular signs and associated systemic conditions were noted. If the active inflammation was present in one eye it as considered unilateral if found in both eyes it was considered bilateral. It was considered acute inflammation if the symptoms were present for less than three months or else it was considered chronic and considered recurrent if two episodes were separated by a disease-free period. Anterior uveitis diagnosed as granulomatous if large keratic precipitates, Koeppe nodules, Busacca nodules were found. A differential diagnosis was made in each case and based on this laboratory investigations were planned. The investigations included complete blood picture, ESR [Erythrocyte Sedimentation Rate], Urine and stool examination, Mantoux test if required, serological tests for syphilis, [Human Immunodeficiency Virus] HIV, rheumatoid factor was performed in all cases. [Chest X-ray] CXR of the chest, lumbosacral area, and knee joints were obtained. All patients were treated medically with topical steroids (prednisolone acetate 1\%) and topical cycloplegic mydriatics (atropine or homatropine). Steroids frequency was titrated according to the severity of uveitis.

Appropriate treatment was given whenever etiology was known. Systemic antimicrobials were prescribed when infectious agents were found to be the cause of uveitis. Administration of systemic steroids was considered in patients where there was severe inflammation and not responding to the treatment or patients with macular edema. Cases in which there was lens-induced inflammation were treated by surgery. If the uveitis was associated with visually significant cataract, Cataract Surgery was performed 3 months after active inflammation subsided. These patients were given high doses of topical and systemic steroids 1 week before operative procedure and tapered thereafter. Those cases of anterior uveitis presenting with secondary glaucoma were given T. ${ }^{11,12}$ Acetazolamide $250 \mathrm{mg}$ thrice daily along with Timolol $0.5 \%$ eye drops twice daily in some cases topical steroids were prescribed. The follow up of cases was done up to six months to evaluate the outcome of treatment and complications. The data obtained were then analysed on Windows format and the statistical analysis was performed by IBM [International Business Machines] SPSS Statistics 20 version. Categorical variables are described as frequency, percentage, and Chi-square test for $\mathrm{p}$ values $(\mathrm{p}<0.05$ was considered significant).

\section{RESULTS}

\section{Demographic profile}

The most common age group in our study was 41-51 years with $n=16(32 \%)$ of cases followed by $n=11(22 \%)$ in the age group 51- 60 years. The mean age of the cases in the study was 47.5 years. More cases were detected in males as compared to females. The male to female ratio was roughly $2: 1$ shown in table 1 . 
Table 1: Demographic profile of patients included in the study

\begin{tabular}{lcccc} 
Age group & Male & Female & $\begin{array}{c}\text { Total Number } \\
(\mathbf{n})\end{array}$ & Percentage \\
$21-30$ & 4 & 1 & 5 & 10 \\
$31-40$ & 7 & 3 & 10 & 32 \\
$41-50$ & 10 & 6 & 16 & 20 \\
$51-60$ & 6 & 5 & 11 & 22 \\
$61-70$ & 5 & 3 & 8 & 16 \\
Total & 32 & 18 & 50 & 100 \\
\hline
\end{tabular}

In the present, the predominant side involved with anterior uveitis was the right eye which included $n=27(54 \%)$ of cases and left eye in $\mathrm{n}=20(40 \%)$ of cases and both eyes were simultaneously involved in $n=3(6 \%)$ of cases shown in figure 1 .

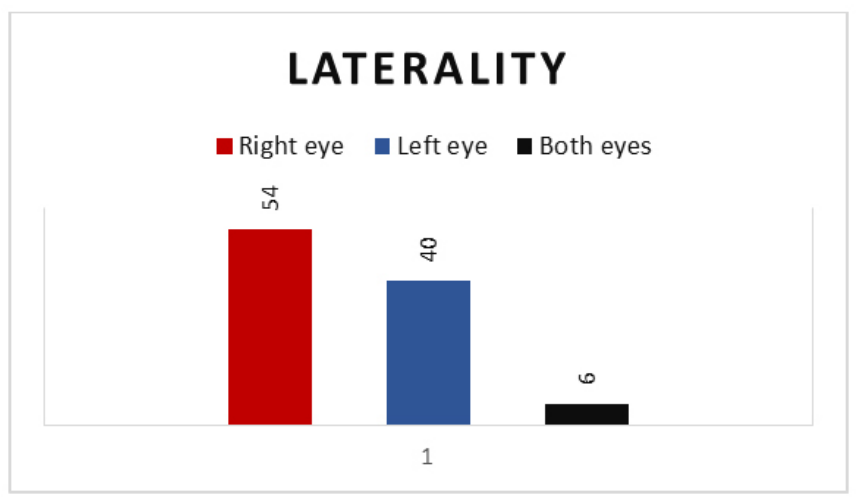

Figure 1: Showing the laterality of involvement of eye in the cases.

\section{Pattern of Uveitis}

In the present study, the pattern of anterior uveitis was studied which showed that most of the patients $n=44(88 \%)$ of the total $n=50(100 \%)$ patients were having acute anterior uveitis and $n=6(12 \%)$ were having a chronic type of anterior. In the present study, most of the cases $\mathrm{n}=45(90 \%)$ were suffering from non-granulomatous infection and $n=5(10 \%)$ were having granulomatous inflammation show in table 2 . The percentage of granulomatous and non-granulomatous uveitis detected in our study is based only on the clinical picture and not by histopathological examination. The study showed the presence of iris nodules (Koeppe's nodules) in cases of chronic anterior uveitis with tuberculosis as the etiology.

Table 2: Showing the Type of Inflammation

\begin{tabular}{lcc} 
Type of Inflammation & \multicolumn{1}{c}{ Cases } \\
& No $(\mathbf{n}=\mathbf{5 0})$ & $\%$ \\
\hline Chronic & 6 & 12 \\
Acute & 44 & 88 \\
Non-granulomatous & 5 & 10 \\
Granulomatous & 45 & 90
\end{tabular}

There was a systemic association present in $n=10(20 \%)$ of the cases of the study and systemic association remained absent in $n=40(80 \%)$ of the cases of the present study. Most of the cases the etiology remained obscure hence kept under the idiopathic section. A few cases were associated with Spondyloarthropathy. The association with Tuberculosis was found in $10 \%$ of cases. The other details of the diagnosis of the cases in the study are shown in table 3 . Anterior uveitis was found to be associated with diabetes mellitus in 3 patients $(6 \%)$ and hypertension in two (4\%) patients (Table 3). All those who had diabetes mellitus were above 50 years of age. One out of five diabetes mellitus patients had chronic uveitis. In a study of uveitis presenting in the older age group, it was found that diabetes should be considered as one of the risk factors for uveitis development. Visual acuity score of 6/12 or lesser was found in the majority of $(87.3 \%)$ of cases at presentation.

Table 3: The Final Diagnosis of the cases of the study

\begin{tabular}{lcc} 
Final Diagnosis & \multicolumn{2}{c}{ Cases } \\
& No $(\mathbf{n = 5 0})$ & $\%$ \\
\hline Idiopathic & 27 & 54 \\
Spondyloarthropathy associated & 65 & 12 \\
Herpes Simplex Keratouveitis & 1 & 2 \\
Tuberculosis & 5 & 10 \\
Fuch's heterochromic uveitis & 1 & 2 \\
Hansen's uveitis & 2 & 4 \\
Lens induced uveitis & 5 & 10 \\
Herpes zoster & 2 & 4 \\
Sclerokerato uveitis & 0 & 0 \\
Inflammatory bowel disease & 1 & 2 \\
Total & 50 & 100 \\
\hline
\end{tabular}

\section{Complications of Uveitis}

Cataract was the common compilation of anterior uveitis out of the $n=7$ cases in this study(Table 4 ). $N=6$ cases reached the maximum post-operative vision of $6 / 12$ and one was able to reach $n=6 / 9$ with refraction and they were prescribed glasses. Cystoid macular edema was treated with topical and subtenon corticosteroids and oral acetazolamide. One improved to one line and second to two lines in the Snellen chart after treatment. One patient of glaucoma was on medical therapy and intraocular pressure controlled and visual field were satisfactorily shown in table 5 .

Table 4: Presenting complications of anterior uveitis in cases of the study

\begin{tabular}{lcc} 
Complication & No of cases & Percentage \\
Cataract & 7 & 14 \\
Cystoid macular edema & 2 & 4 \\
Glaucoma & 1 & 2 \\
Exudative retinal detachment & 0 & 0 \\
Vitreous haemorrhage & 0 & 0 \\
\hline
\end{tabular}


Table 5: Visual acuity before and after treatment of cases with complications

\begin{tabular}{|c|c|c|c|c|c|c|}
\hline \multirow[t]{2}{*}{$\begin{array}{l}\text { Visual } \\
\text { Acuity }\end{array}$} & \multicolumn{2}{|c|}{ Cataract } & \multicolumn{2}{|c|}{$\begin{array}{l}\text { Cystoid Macu- } \\
\text { lar Edema }\end{array}$} & \multicolumn{2}{|c|}{ Glaucoma } \\
\hline & Pre op & Post op & Pre op & Post op & Pre op & Post-op \\
\hline $6 / 60$ & 1 & & & & & \\
\hline $6 / 36$ & 2 & & 02 & & o1 & \\
\hline $6 / 24$ & 2 & & & 01 & & o1 \\
\hline $6 / 18$ & 3 & & & 01 & & \\
\hline $6 / 12$ & & o6 & & & & \\
\hline $6 / 9$ & & 01 & & & & \\
\hline
\end{tabular}

\section{DISCUSSION}

The annual incidence of anterior uveitis in calculated in our study was 15.6 per 100000 patients. The incidence of uveitis in developed countries is been estimated between 17 and 52 per 100000 of the population per year. ${ }^{11}$ Although the incidence in the study appears to be low however, there are chances of less reporting of cases in our poor tribal area and some also get treated by home remedies and quacks as well. ${ }^{13,14}$ It has been estimated that $50 \%$ of patients with uveitis will develop complications and one-third of them suffer from visual impairment and $5-10 \%$ will be blinded due to the disease. ${ }^{11}$ The most common age group in our study was 41 51 years with $n=16(32 \%)$ of cases followed by $n=11(22 \%)$ in the age group 51- 60 years. The mean age of the cases in the study was 47.5 years. Hussain SMP et al. ${ }^{12}$ in Hyderabad found the people with $40-50$ years ( $4^{\text {th }}$ decade) are found to be more prone to anterior uveitis they found $27.53 \%$ of patients in $4^{\text {th }}$ decade with anterior uveitis. Singh et al. have also reported anterior uveitis in the 4th decade of life. ${ }^{13}$ In a similar study by Biswas et al. in Chennai found the incidence of uveitis in the 5th decade of life. ${ }^{14}$ Similarly other studies have also noted that uveitis is more common among the 40-50 years age group. ${ }^{5,15}$ Anterior uveitis is less common under 10 years of age and above 60 years of age. ${ }^{5}$ Our study showed the incidence of anterior uveitis is more common in males as compared to females. Studies done in these areas by other researchers have also shown male preponderance for anterior uveitis. ${ }^{5,13,14,16}$ The right eye which included 27 (54\%) of cases and left eye in $20(40 \%)$ of cases and both eyes most of our cases had unilateral involvement. Rathinam et al. have reported that $85.3 \%$ of patients in their study had a unilateral presentation of anterior uveitis. ${ }^{5,17}$ Although both right and left eye has an equal predilection for the disease. In this study, $88 \%$ of cases were of acute anterior uveitis and $12 \%$ of cases of chronic uveitis. Sudha et al. ${ }^{6}$ reported $75.86 \%$ of acute anterior uveitis and $17.82 \%$ had chronic, and $6 \%$ had recurrent uveitis. In the current study, no specific etiology was found in 54\% of cases arthritis associated were $12 \%$ and tuberculosis was found in $10 \%$ of cases. Sin- gh et al. ${ }^{17}$ in their study found specific etiology of uveitis in $48.2 \%$ of cases and the most common cause detected was tuberculous in $22.16 \%$ cases followed by syphilis in $3.77 \%$ cases. Das et al. ${ }^{18}$ studying patterns of referral in tertiary care reported collagen disorders in $29.4 \%$ of the cases of anterior uveitis. Rathinam et al. ${ }^{5}$ found leptospiral uveitis as the common etiology for uveitis in their cases. It is seen that tuberculosis can involve both anterior as well as the posterior segment of the eye. The typical presentation is a granulomatous type but sometimes maybe non-granulomatous in rare cases. There can be the presence of nodular lesions on the iris and angle of the anterior chamber. It is suspected based on the clinical findings and poor response to steroids and recurrence after stopping steroids and pigmented hypopyon and early neovascularization of iris. Such cases are managed by the administration of standard antitubercular drugs duration of which is 6-9 months. In this study, we found $90 \%$ cases non-granulomatous anterior uveitis and $10 \%$ were the granulomatous types. Sudha et al. ${ }^{6}$ reported non-granulomatous uveitis in $90 \%$ of cases and only $10 \%$ of patients had granulomatous uveitis agreeing with the results of this study. Uveitis is associated with ocular complications such as cataract, glaucoma, band keratopathy, macular edema, epiretinal membrane, and a painful blind eye. In this study, complications were seen in $20 \%$ of cases. Most of them were in chronic cases.

Cataract was seen in $14 \%$ cystoid macular edema in $4 \%$ and glaucoma in $2 \%$ cases. Rothova et al. ${ }^{19}$ reported cataracts in $19 \%$ cases, glaucoma $11 \%$ cases, and phthisical eye in $2.4 \%$ cases. Cataract formation in uveitis is usually developed by uncontrolled prolonged inflammation and long-term use of high doses of topical as well as systemic steroids. All the cases of cataract underwent cataract extraction and PMMA [Polymethyl methacrylate] intraocular lens and the best correction was 6/9 in one case and rest all the cases corrected to $6 / 12$. The long-term ocular inflammation and infections can lead to cystoid macular edema (CME) the common cause is the inflammatory mediated breakdown of the blood-retinal barrier. $^{20}$

In this study there were two cases of CME that were treated with topical and sub-tenon corticosteroids and oral acetazolamide. One improved to one line and second to two lines in the Snellen chart after treatment. There was one case of uveitic glaucoma and was treated with medical therapy and responded well with IOP [Intra Ocular Pressure] within the normal range. Filtration is indicated when there is no response to medical treatment.

\section{CONCLUSION}

The study revealed that the overall incidence of anterior uveitis is lesser in this area probably due to non-reporting by the 
tribal people who are often ignorant of the complications of the disease. The common age group of the presentation was $4^{\text {th }}$ decade and acute non-granulomatous uveitis was found in up to $90 \%$ of cases. In most of the cases, the etiology remained obscure hence included in the idiopathic group. A thorough evaluation and management were done in all cases including treating the associated systemic disorders. Cataract cases were treated with cataractomy and all the cases had acceptable visual acuity post-operation. Early detection and treatment of cases could prevent long term complications and reduce the burden of blindness from this disease.

\section{Source of Financial support: Nil}

\section{Conflict of interest: None}

Ethical Permission: Obtained

\section{ACKNOWLEDGEMENT}

Authors wish to thank the staff of the Department of Ophthalmology, Rajiv Gandhi Institute of Medical Sciences [RIMS] Adilabad for their support during the conduction of the study. The authors are also grateful to authors/editors/ publishers of all those articles, journals and books from where the literature for this article has been reviewed and discussed

\section{REFERENCES}

1. Rothova A, Suttorp-van Schulten MS, Frits Treffers W, Kijlstra A. Causes and frequency of blindness in patients with intraocular inflammatory disease. Br J Ophthalmol 1996; 80(4):332-36.

2. Suttorp-Schulten MS, Rothova A. The possible impact of uveitis in blindness: a literature survey. Br J Ophthalmol 1996;80(9): 844-48.

3. Chang JH, Wakefield D: Uveitis: a global perspective. Ocul Immunol Inflamm 2002; 10(4):263-79.

4. Chowdhuri SK, Jana S. Childhood ocular emergencies: 5 years retrospective clinical audit in a rural medical college and hospital. Int J Curr Res Rev 2013;5(20):116-20.
5. Rathinam SR, Namperumalsamy P. Global variation and pattern changes in the epidemiology of uveitis. Indian J Ophthalmol 2007; 55 (3):173-83.

6. SudhaMadhavi KM, Kumaraswamy RC. Study of clinical and etiological pattern of anterior uveitis in middle Karnataka. CRISMED J Helt Res 2015; 2(2):124-28.

7. Mathur G, Biswas J. Systemic associations of anterior uveitis in a tertiary care ophthalmic center in south India. Int Ophthalmol 2012;32(5):417-20.

8. Rosenbaum JT, Lin P, Asquith M. Does the microbiome cause B27 - related acute anterior uveitis? Ocular Immunol Inflamm 2016;24(4):440-44.

9. Gupta V, Gupta A, Rao NA. Intraocular tuberculosis-an update. Surv Ophthalmol 2007; 52:561-87.

10. Majumder PD, Ghosh A, Biswas J. Infectious uveitis: An enigma. Middle East Afr J Ophthalmol 2017;24(1):2-10.

11. London NJS, Rathinam SR, Cunningham ET. The epidemiology of uveitis in developing countries. Int Ophthalmol Clin Spring 2010;50(2):1-17.

12. Hussain SMP, Mirza A. A prospective study of the clinical profile of patients presenting with anterior uveitis. J Evolution Med Dent Sci 2016;5(99):7280-83.

13. Singh R, Gupta V, Gupta A. Pattern of Uveitis in a referral eye clinic in North India. Indian J Ophthalmol 2004; 52:121-25.

14. Biswas J. Anatomy of the Uveal tract. In: Dutta LC, Dutta NK, Editors Modern Ophthalmology. $3^{\text {rd }}$ Edn. Vol3 New Delhi: Jaypee Brothers; 2005;1249-53.

15. Dogra M, Singh R, Agarwal A, Sharma A, Singh SR, Gautam $\mathrm{N}$, et al. Epidemiology of Uveitis in a Tertiary-care Referral Institute in North India. Ocul Immunol Inflamm. 2017;25(sup1): S46-S53.

16. Gautam N, Singh R, Agarwal A, Yangzes S, Dogra M, Sharma A, et al. Pattern of Pediatric Uveitis at a Tertiary Referral Institute in North India. Ocul Immunol Inflamm 2018;26(3):379385 .

17. Singh R, Gupta V, Gupta A. Pattern of Uveitis in a referral eye clinic in North India. Indian J Ophthalmol 2004; 52:121-25.

18. Das D, Biswas J, Ganesh SK. Pattern of uveitis in a referral uveitis clinic in India. Indian J Ophthalmol 1995; 43:117-21.

19. Rothova A, Suttorp-van Schulten MS, Frits Treffers W, Kijlstra A. Causes and frequency of blindness in patients with intraocular inflammatory disease. Br J Ophthalmol 1996; 80(4):332-36.

20. TG Rotsos, MM Moschos. Cystoid macular edema. Clin Ophthalmol 2008; 2(4): 919-30. 